\title{
Local Antibiotic Therapy for Osteomyelitis of a Replanted Hand
}

\author{
Runa Minami and Naoki Nishijima
}

\begin{abstract}
Background: Although systemic antibiotic therapy is recommended for the prevention and treatment of infection, the use of local antibiotic(s) in the setting of osteomyelitis is well established. Treatment consists of debridement and placement of antibiotics that have been added to bone cement. Hand osteomyelitis will fail periodically after treatment with systemic antibiotics. Debridement is difficult because the fine architecture of the hand limits excessive debridement that might be used in other locations. In the current study, we injected antibiotics directly into the infected bone and soft tissue and had a good result.

Case Presentation: A 65-year-old man had a traumatic amputation and replantation of his right hand. He lost the majority of the skin coverage of the dorsum of the amputated hand. During treatment, his residual extensor tendons and bones had severe methicillin-resistant coagulase-negative staphylococcal (MRCNS) infection. Daily drainage and systemic antibiotics for five months did not resolve the issue. A groin flap and latissimus dorsi flap were insufficient to control the infection. We injected antibiotics every day to the infected region for one month. Methicillin-resistant coagulase-negative staphylococcal infection was undetectable thereafter.
\end{abstract}

Conclusion: Infiltration of local antibiotic therapy is an option when systemic antibiotic therapy fails.

A LTHOUGH SYSTEMIC ANTIBIOTIC THERAPY is recommended for the prevention and treatment of infection, the use of local antibiotic for osteomyelitis is well established. Treatment including debridement, removal of any implant, placement of antibiotic-enhanced bone cement, and wellvascularized tissue flaps are effective in most cases. Antibiotic and delivery system selection is important. Vancomycin and gentamicin are often selected because they have broad spectrum of efficacy, low rate of resistant bacteria including methicillinresistant species, and thermal stability [1-3]. Bone cement is used commonly as an antibiotic carrier. This material can be molded as a transient implant for arthroplasty with beads to fill the dead space. Antibiotic-laden bone cement releases the antibiotic constantly to the infected cavity without raising serum concentration and causing systemic toxicity. The disadvantage of this technique is that bone cement is not biodegradable and has to be removed at the second surgery, it is difficult to mix large amounts of antibiotic into the cement, and antibiotic concentration decreases in a few weeks.

In cases of hand osteomyelitis, thorough debridement is not possible because of the fine architecture of the hand.
Several bones can be totally infected but difficult to sacrifice. Local antibiotic injection is not an accepted treatment but may be of use when other treatments have failed.

\section{Case Presentation}

A 65-year-old man was transferred to our hospital because his right hand had been amputated at the metacarpal level by a mechanical device. There was no history of diabetes mellitus, hypertension, or an immunocompromised condition; he smokes 20 cigarettes per day. On close examination, his thumb had disarticulation at the inter-phalangeal (IP) joint, the second and third metacarpal bones were amputated, and the fourth and fifth finger had disarticulation at the carpometacarpal (CM) joint. The distal part of the second to fifth fingers remained connected only by flexor tendons. Only the thumb had a viable blood supply. The skin was degloved from the wrist to the base of the phalanges. The skin was contaminated with soil that was difficult to remove mechanically from the injury site.

We performed arthrodesis of the thumb IP joint and fourth and fifth finger $\mathrm{CM}$ joint, second and third finger metacarpal

Tango Central Hospital, Kyotango, Kyoto, Japan.

(c) Runa Minami and Naoki Nishijima, 2016; Published by Mary Ann Liebert Inc. This Open Access article is distributed under the terms of the Creative Commons License (http://creativecommons.org/licenses/by/4.0), which permits unrestricted use, distribution, and reproduction in any medium, provided the original work is properly credited. 
bone fixation, and artery revascularization to the palmar arch. We also performed a groin flap to cover the dorsum of his hand. We left most of the avulsed extensor tendons under the groin flap so that reconstruction could be achieved at a second operation. One week after the primary procedure, the remaining skin of dorsum of the MP joint became necrotic secondary to a methicillin-resistant coagulase negative staphylococcal (MRCNS) infection. We treated this with daily drainage and systemic cefazilon, cefotiam, and finally with amikacin to which MRCNS was susceptible. Forty days after replantation, we performed a free latissimus dorsi flap, including end-to-side anastomosis of thoracodorsal artery to ulnar artery and an end-to-end anastomosis of thoracodorsal vein to basilic vein. We achieved good skin coverage with viable flap, but the underlying metacarpal bones and basal phalanges had osteomyelitis and extensive suppurative infection involved the remnant extensor tendons. For the next month we did not prescribe antibiotics but continued drainage and application of gentamicin gel. The infection continued and we started systemic minocycline, cefazolin, cefotiam, and ampicillin-sulbactam, to which MRCNS was initially susceptible but became resistant. Finally we tried daily local injections of amikacin $200 \mathrm{mg}$ for one week and the infected site became culture-negative. We added another week of local injection of amikacin and two weeks of $100 \mathrm{mg}$ of arbekacin. We followed with 10 months without antibiotics; the patient has had debris from a small cutaneous fistula but never had a positive culture from this site.

\section{Discussion}

Replantation failure rate is high in patients with crush and avulsion injuries compared with saw and guillotine injuries [4]. Because of post-operative osteomyelitis and soft tissue infection, this patient's joints were ankylosed and his hand function severely impaired. Retrospectively, there were some things we could have done to prevent post-operative infection. First, the extent of the trauma was under-estimated and debridement was inadequate for the severely contused soft tissue, especially on the dorsum of the MP joints. This caused groin flap necrosis. Second, we left avulsed extensor tendons that were still contaminated with soil under the groin flap for later reconstruction. We washed out the soil thoroughly, but avascular tendons are easily inoculated with bacteria. Third, we could have injected prophylactic aminoglycoside at the time of the first surgery [5]. The incidence of infection of the wound in patients who have an open fracture correlates directly with the extent of soft tissue damage, classified as Gustilo-Anderson fracture type [6]. For type I fractures, the rate of infection ranges from $0 \%-2 \%$; for type II, from $2 \%-$ $7 \%$; for type III, from $10 \%-50 \%$ with a rate of amputation of $50 \%$ or more. Some studies suggest that local administration of aminoglycoside as an adjunct to systemic antibiotic is effective in lowering rates in open fractures.

Amputation with the subsequent use of a prosthetic device is another option to restore hand function. Harness-controlled hand prosthetics and myoelectric hand prostheses may be more useful than an ankylosed hand. In the literature, many authors suggest that replantation of a traumatically amputated arm leads to higher satisfaction rates than a prosthesis, regardless of the objective functional outcome. Sensation and psychological well-being seem to be the two major advan- tages of replantation over a prosthesis [7]. The patient and the surgeon did not choose revision amputation with prosthetic fitting, but rather made an effort to save the hand to minimize emotional trauma.

\section{Conclusion}

Hand osteomyelitis after replantation is sometimes difficult to control using systemic antibiotic because of inadequate blood supply and debridement. Groin flap and latissimus dorsi flaps can provide good skin coverage but in this case was not adequate to control the infection. Local daily injection of aminoglycoside is easy and economical treatment after failure of systemic treatment.

\section{Author Disclosure Statement}

No competing financial interests exist.

\section{References}

1. Hake ME, Young H, Hak DJ, et al. Local antibiotic therapy strategies in orthopaedic trauma: Practical tips and tricks and review of the literature. Injury 2015;46:1447-1456.

2. Nandi SK, Mukherjee P, Roy S, et al. Local antibiotic delivery systems for the treatment of osteomyelitis-A review. Mater Sci Eng C 2009;29:2478-2485.

3. Gogia JS1, Meehan JP, Di Cesare PE, et al. Local antibiotic therapy in osteomyelitis. Semin Plast Surg 2009;23:100-107.

4. He JY, Chen SH, Tsai TM. The risk factors for failure of an upper extremity replantation: Is the use of cigarettes/tobacco a significant factor? PLoS One 2015;10:e0141451.

5. Lawing CR, Lin F-C, Dahners LE. Local injection of aminoglycosides for prophylaxis against infection in open fractures. J Bone Joint Surg Am 2015;97:1844-1851.

6. Gustilo RB, Merkow RL, Templeman D. The management of open fractures. J Bone Joint Surg Am 1990;72:299-304.

7. Otto IA, Kon M, Schuurman AH, et al. Replantation versus prosthetic fitting in traumatic arm amputations: A systematic review. PLoS One 2015;10:e0137729.

Address correspondence to:

Dr. Runa Minami

Tango Central Hospital

Mineyama Sugitani 158-1

Kyotango

Kyoto, 627-8555

Japan

E-mail:xxluna_selenaxx@yahoo.co.jp

$\begin{aligned} & \text { Abbreviations Used } \\ \mathrm{IP} & =\text { inter-phalangeal } \\ \mathrm{MP} & =\text { metacarpophalangeal } \\ \mathrm{MRCNS}= & \text { methicillin-resistant coagulase } \\ & \text { negative staphylococci }\end{aligned}$

Cite this article as: Minami R, Nishijima N (2016) Loca antibiotic therapy for osteomyelitis of a replanted hand. Surgical Infections Case Reports 1:1, 126-127, DOI: $10.1089 / \mathrm{crsi} .2016 .0031$ 